\title{
INTENCIÓN EMPRENDEDORA \\ EN ESTUDIANTES UNIVERSITARIOS \\ EN EL CENTRO DE TAMAULIPAS, MÉXICO
}

\author{
MÓNICA LORENA SÁNCHEZ LIMÓN \\ Universidad Autónoma de Tamaulipas, México \\ msanchel@docentes.uat.edu.mx \\ MARIANA ZERÓN FÉLIX \\ Universidad Autónoma de Tamaulipas, México \\ zfmariana@docentes.uat.edu.mx \\ MANUEL HUMBERTO DE LA GARZA CÁRDENAS \\ Universidad Autónoma de Tamaulipas, México \\ mdelagarza@uat.edu.mx.com
}

\section{RESUMEN}

El presente trabajo tiene por objetivo, en primera instancia identificar los factores que determinan la intención emprendedora entre los alumnos de las Universidades en Ciudad Victoria, Tamaulipas, para posteriormente identificar aquellos elementos que inciden positiva y significativamente en dicha intención. Apegándose a la Teoría del Comportamiento Planeado y mediante un análisis factorial exploratorio, se determinan los componentes de la intención emprendedora y, mediante una regresión lineal, se comprueba que la valoración, la formación universitaria y los objetivos empresariales tienen gran incidencia en la intención de emprender. Para dicho trabajo el sujeto de estudio fueron alumnos de carreras económico-administrativas, puesto que son ellos los que desarrollan conocimientos y habilidades formales sobre el área a estudiar.

PALABRAS CLAVE: INTENCIÓN EMPRENDEDORA, TEORÍA DEL COMPORTAMIENTO PLANEADO, RECONOCIMIENTO SOCIAL.

\section{ABSTRACT}

The purpose of this study is to identify the factors that determine the entrepreneurial intention among the students of the universities in Victoria City, Tamaulipas. In order to identify the elements that have a positive and significant impact on that intention. Clinging to the Theory of Planned Behavior and exploratory factor analysis determine the components of the entrepreneurial intention and, through linear regression it is verified that the valuation, the university formation and the business objectives have a great incidence in the intention to undertake. For this work the subject of study were the students of economic-administrative careers who are they who develop formal knowledge and skills about the area to study.

KEYWORDS: ENTREPRENEURIAL INTENTION, THEORY OF PLANNED BEHAVIOR, SOCIAL RECOGNITION. 


\section{INTRODUCCIÓN}

Actualmente, como resultado de un entorno altamente competitivo, las naciones, tanto en su economía como en su política, deben estar preparadas para responder a los cambios del entorno y preservar la estabilidad macroeconómica frente a cualquier contingencia; una de estas acciones puede ser el apoyo a la creación de nuevas empresas (Audretsch y Keilbach, 2004; Aloulou y Aloulou, 2016; Wennekers, van Stel, Thurik y Reynolds, 2005).

Estas unidades económicas creadas aprovechan las oportunidades de negocio, ya que tienden a ajustarse a los movimientos del mercado y a atender los cambios en la demanda (Weiss, 1991; Hayter, 2016; Mills y Schuman, 1985). La detección de oportunidades para la apertura de un negocio se da por el talento o el espíritu emprendedor, ambas propias del individuo (Evans y Leighton, 1990). Sin embargo, el entorno juega un papel relevante en el desarrollo del emprendedor (Bergmann, Hundt y Sternberg, 2016).

En este sentido, de acuerdo con Shane (2004) el emprendimiento, en el ámbito empresarial, incluye elementos como la identificación y evaluación de oportunidades, los esfuerzos para obtener recursos y el desarrollo de la estrategia, entre otros (Forsström, Jussila y Kolhinen, 2015). Las nuevas empresas generan una gran parte de la tasa del crecimiento del empleo de los países y, además de contribuir a la producción nacional son una poderosa fuente de innovación, (Amit, Glosten y Muller, 1993; Díaz, 2002).

El estudio del emprendimiento es de tal importancia que se han creado programas como el Global Entrepreneurship Monitor (GEM), el cual es una evaluación anual de los niveles nacionales de la actividad emprendedora. El GEM ha realizado estudios en México de forma regular (2001, 2002, 2005, 2006, 2008, 2010, 2011, 2012, 2013, 2014 y 2015) incluyendo en los últimos años estudios regionales.
Dentro de los principales resultados en México, se aprecia la tasa de creación de empresas ya que se duplicaron de un 10,5\% en 2010 a 21\% de la población en 2015; la tercera parte de los emprendedores cuentan con secundaria solamente y existe una diferencia de género ya que los hombres perciben mayores oportunidades de negocio, se sienten más competentes y confían en su capacidades para emprender (Hernández, González y Herrera, 2015).

Se puede concluir que los resultados son positivos, ya que existe un crecimiento en los niveles de actividad emprendedora como una mejora en los factores del entorno emprendedor (Edelman, Manolova, Shirokova y Tsukanova, 2016; Martin, McNally y Kay, 2013). Además, cada vez existe mayor evidencia de que el ambiente es esencial para el desarrollo emprendedor; en este sentido, las universidades juegan un rol importante, puesto que inciden en las personas en cuanto a la habilidad de detección de oportunidades (Bergmann et al., 2016; Hoppe, 2016).

El aumento de emprendedores universitarios deriva, en cierta forma, de la falta de inserción en el mercado laboral al egresar de la institución de educación superior (Edelman et al., 2016) lo cual indica un rango mayor de jóvenes emprendedores como respuesta al nivel de desempleo en recién egresados (Pérez, González y Rodríguez, 2016). En consecuencia las instituciones de educación superior fomentan en sus alumnos los conocimientos, actitudes y competencias emprendedoras (Karimi, Biemans, Lans, Chizari y Mulder, 2016).

Por lo que se plantea cuáles son los factores que determinan el emprendimiento de los jóvenes universitarios. Para responder a esta pregunta, se establecen dos objetivos; en primera instancia, se busca identificar los factores que determinan la intención emprendedora, para posteriormente comparar los niveles de emprendimiento entre las Universidades en Ciudad Victoria. 
Para poder cumplir con lo anterior, este trabajo se estructura de la siguiente manera: posterior a la introducción, se incluye una revisión de la literatura que ayuda a definir las hipótesis a comprobar en esta investigación; al finalizar, se incluye el apartado metodológico, que explica el método a seguir; en el cuarto apartado, se exponen los resultados del estudio empírico, que son utilizados en el apartado de conclusiones para discutir los hallazgos; y finalmente, se anexa la bibliografía utilizada.

\section{REVISIÓN DE LA LITERATURA}

El término emprendedor fue introducido en la bibliografía económica por Richard Cantillon en 1755 en su obra Essai sur la Nature du Commerce en Général para identificar a quienes corrían el riesgo y tomaban la responsabilidad de poner en marcha y llevar a término un proyecto innovador (Cantillon, 2001).

Siguiendo el desarrollo del concepto, Díaz (2002) diferencia el concepto de emprendedor y el de empresario, ya que el primero cuenta con una aceptación y el segundo no. Sin embargo, una de las principales características es la alta aceptación del riesgo, además de que su comportamiento va dirigido a crear unidades de negocio ante la ausencia de elementos que satisfagan ciertas necesidades (Davids, 1963; Gartner, 1988; McClelland, 1961), desde una perspectiva de innovación (Schumpeter, 1934).

En las últimas décadas ha existido un auge en el estudio del emprendimiento, dicho fenómeno se aborda desde el enfoque económico, donde se imponen los aspectos relativos a la conducta racional y plantea que la actividad emprendedora solamente obedece a cuestiones de carácter económico (Audretsch y Keilbach, 2004; Wennekers et al., 2005).

También se ha estudiado desde el enfoque psicológico, el cual postula que es la individualidad, mediante los rasgos psicológicos de las personas, lo que determina el emprendimiento (Collins, Moore y Unwalla, 1964; McClelland, 1961; Carsrud y Johnson, 1989). Así mismo, desde el enfoque sociológico o institucional que argumenta que es el entorno socio-cultural el que condiciona y propicia la decisión de crear una empresa (Shapero y Sokol, 1982; Steyaert y Katz, 2004).

Por otra parte, un aspecto que resalta dentro del emprendimiento es explotar alguna oportunidad; el proceso, complejo, inicia en la detección de oportunidad y culmina con la implementación del proyecto (Shane y Eckhardt, 2003). A partir de esto se puede apreciar que el conocimiento, que es un atributo de la persona y la información que tiene, es esencial para la explotación de la oportunidad (Edelman et al., 2016).

Lo anterior indica que, de acuerdo con el conocimiento previo con el que cuente el emprendedor podrá utilizarlo a su favor y aumentar sus capacidades de encontrar esa oportunidad y aprovecharla para obtener beneficios (Bergmann et al., 2016). Además, muchos de los rasgos que han sido estudiados incluyen, ambición, necesidad de logro, toma de riesgos, locus de control (Brockhaus, 1982; Casson, 1982), tolerancia a la incertidumbre y ambigüedad, comprensión de la realidad y visión, así como los valores y estilo de toma de decisiones (Gartner, 1988).

El autoempleo o trabajo por cuenta propia puede ser considerado como la forma más simple de emprendimiento, como vía alterna y atractiva a la condición de asalariado (Fernández y Romero, 2013). Los resultados empíricos obtenidos por Hamilton (2000) sugieren que los beneficios no económicos del autoempleo son sustancialmente más importantes para los emprendedores. Convertirse en su propio jefe es el mayor beneficio para ellos. Además, la creación de nuevos negocios dentro de una empresa existente (filiales, joint-ventures, spin.offs), también es considerado emprendimiento (Díaz, 2002; Nueno, 1994). 
Los emprendedores habituales aprenden de las experiencias anteriores, de tal manera que van adquiriendo una tecnología de creación de empresas (Boh, De-Haan y Strom, 2016). Los emprendedores habituales cuentan con cierto conocimiento acumulado, ya que las habilidades adquiridas en ocasiones se transfieren y aplican a los siguientes procesos de creación de nuevas empresas (Martin et al., 2013; MacMillan, 1986).

La necesidad de crear y desarrollar empresas ha animado a los gobiernos regionales, universidades, cámaras de comercio, empresas privadas, o incluso a organizaciones no gubernamentales, al establecimiento y desarrollo de incubadoras. De ahí que las instituciones educativas, como incubadora de spinoffs, se ha convertido en uno de los lugares idóneos para crear empresas (Shane, 2004).

Como se ha comentado, previo a la explotación de la oportunidad o la concreción material de la idea, es necesario tener detectada una oportunidad y, posteriormente, la intención de emprender acciones para ella, considerada como el elemento predictor más importante del comportamiento, es decir, la acción de emprender (Ajzen, 2002).

Para poder analizar mejor la intención se necesitan modelos que nos permitan explicar por qué realmente se toma la decisión y actúan las personas; dado que la intención emprendedora puede verse afectada por diversos factores como lo son necesidades, valores, deseos, hábitos y creencias (Bird, 1988; Lee y Wong, 2004).

Asimismo, existen factores externos que influyen en las actitudes hacia el espíritu empresarial, tales como la limitación de tiempo, dificultad de tarea y la influencia de otras personas a través de la presión social (Lee y Wong, 2004). Además de las variables cognitivas que influyen en la intención, están las de motivación; cuando más favorables sean estas, aumentará la puesta en marcha de la intención (Liñan, 2004).
Para ello, la teoría del comportamiento planeado de Ajzen (1991) menciona que un individuo basa su comportamiento a partir de sus intenciones, relacionadas con la actitud hacia el comportamiento, las normas subjetivas y el control que percibe; lo anterior incide en la convicción de la persona para realizar algo o no (Staniewski y Awruk, 2016).

Posteriormente, Shapero (1982) desarrolló su modelo, llamado Entrepreneurial Event Model (EEM), el cual se centra en características humanas intrínsecas como la experiencia, capacidades, autonomía y actitud ante el riesgo. La elección para crear de una empresa depende de la percepción de deseabilidad, la percepción de viabilidad y la propensión a actuar.

Por otro lado, según Bandura, Freeman y Lightsey (1999), en su teoría del aprendizaje social y autosuficiencia, un cambio en el comportamiento puede ser producido por diferentes métodos que derivan en un comportamiento mecánico. De esta manera, al contar con una alta eficacia percibida, aumenta el cumplimiento de objetivos, reduce el estrés y disminuye la vulnerabilidad a la depresión (Bandura, 1997).

Es así como la intención emprendedora se define como el grado de confianza que un individuo tiene con respecto a la creación de un nuevo negocio (Engle, Schlaegel y Dimitriadi, 2011). Con base en estas teorías y tomando como base lo planteado por Liñan (2004) se desarrollaron los siguientes aspectos de propensión hacia la creación de empresas:

\section{Planteamiento de hipótesis}

\section{Valoración social}

El individuo percibe la aprobación o desaprobación del entorno para poder realizar una actividad emprendedora. La aprobación o desaprobación puede venir del entorno cercano, como 
lo son familiares y amigos; o del ambiente social, ya sea por la escuela en que estudia o la cultura del país (Edelman et al., 2016). Por lo tanto, la cultura (valores propios de una sociedad) motiva que los individuos se involucren en ciertos comportamientos (Mueller y Thomas, 2001; Villasana, Alcaraz y Álvarez, 2016).

Es así como Hofstede et al. (2004) considera dos formas en que la influencia de la cultura es ejercida. Una es de manera positiva, haciendo que la cultura esté de acuerdo con las instituciones económicas y sociales, logrando un entorno más favorable para la actividad empresarial. Cuando la cultura no crea ese entorno de emprendimiento favorable, la insatisfacción individual hará que se busque la satisfacción personal mediante el auto-empleo. Por lo anterior, proponemos la siguiente hipótesis:

H1.- El reconocimiento social del emprendedor tiene una influencia positiva en la intención emprendedora.

\section{Formación y experiencia}

La formación y experiencia son factores determinantes en el grado de autoeficacia percibida, porque se cuenta con mayor número de herramientas y conocimientos para llevar proyectos a su culminación (Bergmann et al., 2016; Boh et al., 2016; Karimi et al., 2016). El entorno y especialmente la educación superior está asociada con una mayor calidad del emprendimiento (Hoppe, 2016). Por ello incluimos la siguiente hipótesis:

H2.- La educación universitaria fomenta la creación de empresas.

\section{Conocimiento empresarial}

Guerrero y Urbano (2012) señalan la importancia de los contactos empresariales para la buena marcha profesional. La exposición previa del individuo a temas relativos con la iniciación de un negocio incide en su capacidad emprendedora, al aumentar su capacidad y al disminuir el miedo al riesgo (Hayter, 2016).

Del mismo modo, Ajzen (2012) comenta que un mayor conocimiento de aspectos empresariales contribuirá a una percepción más realista acerca de la actividad empresarial; por lo tanto, influirá directamente en las intenciones. Lo anterior está representado en la siguiente hipótesis:

H3.- El conocimiento empresarial tiene una influencia positiva en la intención emprendedora.

\section{Éxito empresarial}

El éxito es un factor relevante para el emprendedor. El retorno de beneficios financieros, además del social, por haber asumido riesgos, lo impulsa a seguir adelante. El haber elegido la opción de no ser asalariado y ser empresario tiene su recompensa (Villasana et al., 2016). En este sentido, las actitudes del individuo rigen la visión del mundo y moderan su comportamiento y la percepción del éxito (Plotnikova, Romero y Martínez, 2016), es decir, una percepción positiva de sus actividades incidirá de forma similar y lo motivará a seguir adelante (Cantner, Goethner y Silbereisen, 2017). Por lo anterior, se propone la siguiente:

H4.- La percepción de éxito tiene una influencia positiva en la intención emprendedora.

\section{Capacidades específicas}

En este elemento, se analiza el grado de confianza que cree tener el individuo para desarrollar una serie de habilidades o capacidades. Lucas, Cooper, Ward y Cave (2009) realizaron un estudio donde un bloque medía las habilidades para ser emprendedor mediante una escala de autoeficacia para ciertas capacidades relacionadas con funciones de gestión emprendedora. El emprendedor encuentra motivación al percibir que obtiene resultados positivos en su organización (Aloulou y Aloulou, 2016; Micozzi, Micozzi, Luca- 
FIGURA 1

MODELO INTENCIÓN EMPRENDEDORA

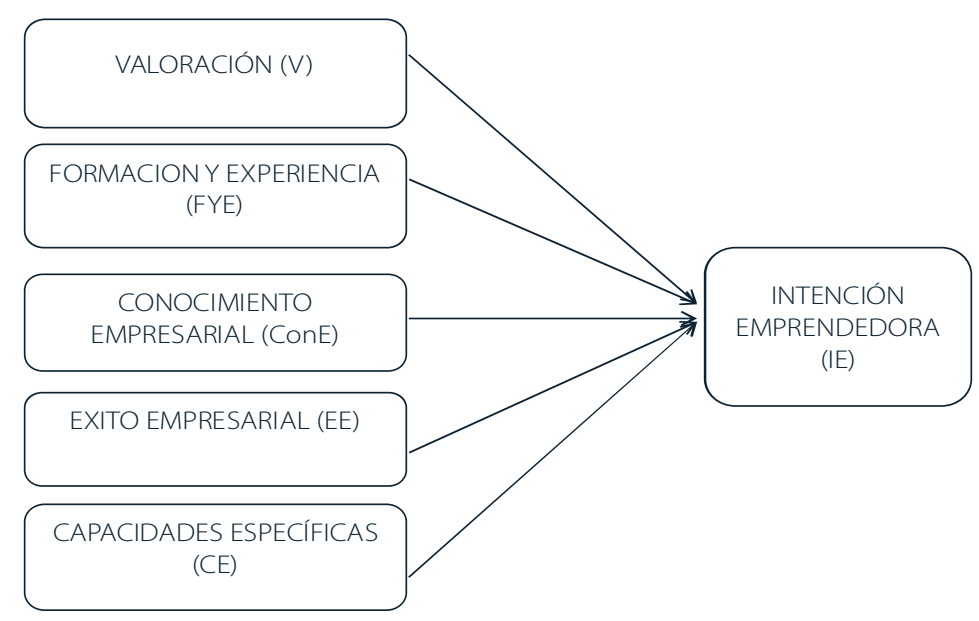

Fuente: United Explanations (2015).

relli y Lucarelli, 2016; Staniewski y Awruk, 2016). Proponemos entonces la siguiente hipótesis:

H5.- La percepción de crecimiento de una empresa fomenta la intención emprendedora.

Lo anterior lo podemos resumir con la figura 1, en la cual se plantea la relación esperada entre las variables.

En la Tabla 1, se resumen los resultados de los estudios empíricos más recientes analizados.

\section{METODOLOGÍA}

Para cumplir con los objetivos planteados, esta investigación se basa en el método cuantitativo para probar las hipótesis establecidas previamente. Asimismo, tendrá un alcance correlacional, puesto que se pretende conocer la relación que existe entre la intención emprendedora y las variables independientes (valoración, capacidades específicas, conocimientos y objetivos empresariales), para lo cual se pondrán a prueba las hipótesis planteadas en el apartado anterior.
El instrumento que se aplicó es con base en Liñan y Chen (2009) y contiene ítems que fueron medidos mediante una escala Likert de siete niveles, en donde 1 es el nivel más bajo (muy en desacuerdo) y 7 el más alto (muy de acuerdo). El cuestionario de intenciones emprendedoras (CIE) está integrado por ochenta y cinco preguntas Likert divididas en nueve bloques.

Para recolectar la información necesaria, se seleccionó una muestra de jóvenes universitarios que cursan carreras de carácter económico-administrativo en la Facultad de Comercio y Administración Victoria (FCAV) y en la Universidad Politécnica de Victoria (UPV). De acuerdo con Bergmann et al. (2016), son ellos los que cuentan con una mayor percepción de contar con capacidades para emprender; asimismo, el nivel de educación es un factor clave, ya que las personas con educación superior muestran una mayor percepción e intención de emprender (Hoppe, 2016; Martin et al., 2013). 
TABLA 1

RESUMEN DE RESULTADOS EMPÍRICOS DE LA REVISIÓN DE LA LITERATURA

\begin{tabular}{|c|c|c|}
\hline Autores & Teorías & Resultados \\
\hline Romero y Milone (2016) & $\begin{array}{l}\text { Teoría del Comportamiento } \\
\text { Planeado }\end{array}$ & $\begin{array}{l}\text {-Hombres, mayor intención, confianza en habilidades y deseo de crear un negocio. } \\
\text { Jóvenes, actitud más positiva al emprendimiento. }\end{array}$ \\
\hline $\begin{array}{l}\text { Arias, Cadavid, Echeverriy } \\
\text { Awad (2012) }\end{array}$ & $\begin{array}{l}\text { Teoría del Comportamiento } \\
\text { Planeado; Modelo del Evento } \\
\text { Emprendedor }\end{array}$ & -Existe fuerte asociación entre conveniencia, tolerancia y viabilidad percibida. \\
\hline Fernández y Gervilla (2013) & $\begin{array}{l}\text { Teoría del Comportamiento } \\
\text { Planeado }\end{array}$ & $\begin{array}{l}\text {-Autoconfianza y valoración de los resul tados son determinantes en la intención } \\
\text { emprendedora. Mujer depende de mayor número de variables para emprender. }\end{array}$ \\
\hline $\begin{array}{l}\text { Brito, Cruz y Hernández } \\
\text { (2014) }\end{array}$ & $\begin{array}{l}\text { Teoría del Comportamiento } \\
\text { Planeado; Modelo del Evento } \\
\text { Emprendedor }\end{array}$ & $\begin{array}{l}\text {-Menor vocación emprendedora de las mujeres. Actitud personal y control percibido } \\
\text { del comportamiento influyen positivamente en la intención emprendedora. }\end{array}$ \\
\hline Mora (2011) & $\begin{array}{l}\text { Teoría del Comportamiento } \\
\text { Planeado }\end{array}$ & $\begin{array}{l}\text {-Emprendedores se consideran competentes ante retos (autoestima), nuevas } \\
\text { perspectivas de realizar las cosas (innovación), orgullo por resultados alcanzados y } \\
\text { satisfacción por sus esfuerzos (motivación al logro), decisiones propias y prevención } \\
\text { de acontecimientos del entorno. }\end{array}$ \\
\hline Osorio y Roldán (2016) & $\begin{array}{l}\text { Teoría del Comportamiento } \\
\text { Planeado; Modelo del Evento } \\
\text { Emprendedor }\end{array}$ & $\begin{array}{l}\text {-La intención emprendedora puede ser explicada por las actitudes personales y la } \\
\text { percepción de control de comportamiento, así como por la norma subjetiva, pero con } \\
\text { un coeficiente bajo. }\end{array}$ \\
\hline Liñan, Nabi y Krueger (2013) & $\begin{array}{l}\text { Teoría del Comportamiento } \\
\text { Planeado }\end{array}$ & $\begin{array}{l}\text {-Valuación cercana y social tienen influencia directa sobre la actitud y norma subje- } \\
\text { tiva. Actitud con mayor efecto en España y el control percibido del comportamiento } \\
\text { en Inglaterra. }\end{array}$ \\
\hline $\begin{array}{l}\text { Durán, Martínez, López, } \\
\text { Flores y Jiménez (2016) }\end{array}$ & $\begin{array}{l}\text { Teoría del Comportamiento } \\
\text { Planeado }\end{array}$ & $\begin{array}{l}\text {-Actitud personal, control percibido y valoración afectan positivamente la intención: } \\
\text { los años no influyen. }\end{array}$ \\
\hline $\begin{array}{l}\text { Riquelme, Ortíz, Álvarez y } \\
\text { Baro (2016) }\end{array}$ & $\begin{array}{l}\text { Teoría del Comportamiento } \\
\text { Planeado }\end{array}$ & $\begin{array}{l}\text {-Con el paso de la carrera no aumenta la intención: solo se muestra con una mejor } \\
\text { valoración del emprendedor. }\end{array}$ \\
\hline $\begin{array}{l}\text { Rachmawan, Lizar y Man- } \\
\text { gundjaya (2015) }\end{array}$ & $\begin{array}{l}\text { Teoría del Comportamiento } \\
\text { Planeado; Modelo del Evento } \\
\text { Emprendedor }\end{array}$ & $\begin{array}{l}\text {-Autoeficacia influye positivamente en la intención emprendedora. Experiencia con } \\
\text { madre emprendedora influye positivamente en la intención emprendedora. }\end{array}$ \\
\hline
\end{tabular}

Fuente: elaboración propia.

El instrumento fue aplicado en julio de 2016. En la FCAV se encontró un total de 149 alumnos inscritos en el octavo semestre de su carrera. Mediante el cálculo de una muestra aleatoria simple, con un nivel de confianza del 95\% (valor $Z$ de 1,96) y un margen de error equivalente a un 5\%, se obtuvo una muestra de 107 alumnos.

Así mismo, en la UPV se contó con una población de 51 alumnos inscritos en el último período de la carrera de Licenciatura en Administración y Gestión de Pymes. Utilizando los mismos parámetros mencionados anteriormente para el cálculo muestral, se obtuvo un total de 45 alumnos a encuestar. Finalmente, se obtuvo un total de 143 encuestas contestadas satisfactoriamente, de las cuales 95 correspondían a alumnos de la FCAV, mientras que las restante 48 procedían de la UPV. 


\section{RESULTADOS}

Se puede ver que la muestra estuvo compuesta en una mayor proporción por mujeres (casi dos tercios de los encuestados). Las carreras con mayor cantidad de alumnos participantes en este estudio fueron Contabilidad Pública y Licenciatura en Administración y Gestión de Pymes.

Se realizó un análisis factorial exploratorio (EPA) como herramienta para identificar y medir las dimensiones adecuadas de la intención emprendedora. Para tal efecto se utilizó el método de componentes principales y rotación varimax. En primera instancia se validó la adecuación muestral mediante la prueba de KMO y se obtuvo un valor de .863. Así mismo, la prueba de esfericidad de Barlett fue significativa ( $p$ valor de .000).
Posteriormente, se determinó el número de componentes resultantes de este análisis, estudiando el total de la variable explicada (ver tabla 3). Con base en ello se determinó seleccionar cinco componentes, al ser éstos los que cumplen con un autovalor mayor a 1; así mismo cumplen con el porcentaje mínimo de la varianza para cada componente (mayor a 2,94\%).

A su vez, mediante la matriz de componentes rotados (ver tabla 4), se estudiaron las cargas factoriales de cada ítem para determinar la composición de cada componente, determinando la existencia del reconocimiento social (valoración), percepción de crecimiento, conocimiento empresarial, percepción de éxito y, educación universitaria (formación y experiencia). De igual forma se validó su fiabilidad mediante el alfa de Cronbach.

TABLA 2

RESUMEN DE RESULTADOS EMPÍRICOS DE LA REVISIÓN DE LA LITERATURA

\begin{tabular}{|c|c|c|}
\hline Estadísticos descriptivos & Teorías & Resultados \\
\hline \multirow[t]{3}{*}{ Género } & Masculino & 36,4 \\
\hline & Femenino & 63,6 \\
\hline & Total & 100 \\
\hline \multirow[t]{5}{*}{ Carrera } & Contador Público & 39,1 \\
\hline & Licenciado en Administración & 19,6 \\
\hline & Licenciado en Informática & 7,8 \\
\hline & Licenciado en administración y gestión de pymes & 33,5 \\
\hline & Total & \\
\hline \multirow[t]{6}{*}{ Nivel de ingreso (mensual, en dólares americanos) } & $0-418$ & 52,3 \\
\hline & $419-836$ & 28,5 \\
\hline & $837-1673$ & 13,5 \\
\hline & $1674-3347$ & 4,3 \\
\hline & Más de 3347 & 1,4 \\
\hline & Total & 100 \\
\hline
\end{tabular}

Fuente: elaboración propia. 
TABLA 3

VARIANZA TOTAL EXPLICADA

\begin{tabular}{|c|c|c|c|c|c|c|}
\hline \multicolumn{4}{|c|}{ Autovalores iniciales } & \multicolumn{3}{|c|}{$\begin{array}{l}\text { Sumas de las saturaciones al cuadrado de la } \\
\text { extracción }\end{array}$} \\
\hline Componente & Total & $\%$ de la varianza & $\%$ acumulado & Total & \% de la varianza & $\%$ acumulado \\
\hline 1 & 12,139 & 35,702 & 35,702 & 12,139 & 35,702 & 35,702 \\
\hline 2 & 3,694 & 10,866 & 46,568 & 3,694 & 10,866 & 46,568 \\
\hline 3 & 2,532 & 7,447 & 54,015 & 2,532 & 7,447 & 54,015 \\
\hline 4 & 2,250 & 6,617 & 60,631 & 2,250 & 6,617 & 60,631 \\
\hline 5 & 1,608 & 4,730 & 65,361 & 1,608 & 4,730 & 65,361 \\
\hline 6 & 970 & 2,854 & 68,215 & & & \\
\hline 7 &, 880 & 2,587 & 70,802 & & & \\
\hline 8 & ,797 & 2,345 & 73,147 & & & \\
\hline 9 &, 759 & 2,232 & 75,379 & & & \\
\hline 10 &, 734 & 2,159 & 77,538 & & & \\
\hline 11 &, 623 & 1,831 & 79,369 & & & \\
\hline 12 &, 597 & 1,754 & 81,124 & & & \\
\hline 13 &, 567 & 1,669 & 82,792 & & & \\
\hline 14 &, 521 & 1,532 & 84,324 & & & \\
\hline 15 &, 510 & 1,499 & 85,823 & & & \\
\hline 16 &, 501 & 1,474 & 87,297 & & & \\
\hline 17 & 446 & 1,311 & 88,609 & & & \\
\hline 18 &, 427 & 1,256 & 89,864 & & & \\
\hline 19 &, 362 & 1,064 & 90,929 & & & \\
\hline 20 &, 343 & 1,008 & 91,937 & & & \\
\hline 21 & 302 &, 888 & 92,825 & & & \\
\hline 22 & ,293 &, 862 & 93,687 & & & \\
\hline 23 & 266 &, 784 & 94,470 & & & \\
\hline 24 & 261 &, 767 & 95,238 & & & \\
\hline 25 & ,245 &, 722 & 95,959 & & & \\
\hline 26 &, 214 &, 630 & 96,589 & & & \\
\hline 27 & 212 &, 623 & 97,213 & & & \\
\hline 28 & ,199 &, 586 & 97,799 & & & \\
\hline 29 & ,171 &, 503 & 98,302 & & & \\
\hline 30 & ,154 &, 454 & 98,756 & & & \\
\hline 31 &, 134 &, 395 & 99,152 & & & \\
\hline 32 & ,119 &, 349 & 99,501 & & & \\
\hline 33 &, 098 & ,289 & 99,790 & & & \\
\hline 34 & ,071 & ,210 & 100,000 & & & \\
\hline
\end{tabular}

Fuente: elaboración propia.

Nota: Método de extracción: Análisis de componentes principales. 
TABLA 4

MATRIZ DE COMPONENTES ROTADOS Y TEST DE FIABILIDAD

\begin{tabular}{|c|c|c|c|c|c|c|c|}
\hline \multirow[t]{2}{*}{ Variable } & \multicolumn{7}{|c|}{ Componente } \\
\hline & Còdigo & 1 & 2 & 3 & 4 & 5 & $\begin{array}{l}\text { Alfa de } \\
\text { Cronbach }\end{array}$ \\
\hline \multirow{8}{*}{$\begin{array}{l}\text { Valoración - El reconocimiento social del empren- } \\
\text { dedor tiene una influencia positiva en la intención } \\
\text { emprendedora. }\end{array}$} & v8 & 0,779 & 0,172 & 0,076 & $-0,125$ & $-0,052$ & \multirow[t]{8}{*}{, 895} \\
\hline & V2 & 0,772 & 0,134 & 0,06 & 0,026 & 0,068 & \\
\hline & v5 & 0,755 & $-0,067$ & 0,179 & 0,149 & 0,056 & \\
\hline & v6 & 0,752 & 0,234 & 0,177 & 0,022 & 0,077 & \\
\hline & v7 & 0,716 & 0,216 & 0,168 & 0,102 & 0,251 & \\
\hline & V3 & 0,694 & 0,121 & 0,227 & $-0,089$ & 0,175 & \\
\hline & v4 & 0,602 & 0,108 & 0,177 & 0,225 & 0,395 & \\
\hline & v1 & 0,509 & 0,307 & 0,049 & 0,139 & 0,406 & \\
\hline \multirow{7}{*}{$\begin{array}{l}\text { Crecimiento Empresarial -La percepción de creci- } \\
\text { miento de una empresa fomenta la intención em- } \\
\text { prendedora. }\end{array}$} & de4 & 0,061 & 0,794 & 0,213 & 0,218 & 0,141 & \multirow[t]{7}{*}{, 909} \\
\hline & de 5 & 0,113 & 0,755 & 0,245 & 0,192 & 0,193 & \\
\hline & de6 & 0,161 & 0,754 & 0,065 & 0,29 & 0,187 & \\
\hline & de2 & 0,162 & 0,715 & 0,297 & 0,14 & 0,144 & \\
\hline & de7 & 0,201 & 0,685 & $-0,035$ & 0,197 & 0,153 & \\
\hline & de3 & 0,198 & 0,672 & 0,307 & 0,097 & 0,091 & \\
\hline & del & 0,247 & 0,626 & 0,264 & 0,377 & 0,137 & \\
\hline \multirow{6}{*}{$\begin{array}{l}\text { Conocimiento Empresarial - El conocimiento empre- } \\
\text { sarial tiene una influencia positiva en la intención } \\
\text { emprendedora. }\end{array}$} & as3 & 0,165 & 0,253 & 0,828 & 0,067 & 0,105 & \multirow[t]{6}{*}{, 919} \\
\hline & as5 & 0,218 & 0,271 & 0,773 & 0,064 & 0,187 & \\
\hline & as6 & 0,292 & 0,187 & 0,761 & $-0,052$ & 0,219 & \\
\hline & as2 & 0,127 & 0,03 & 0,745 & 0,144 & 0,231 & \\
\hline & as1 & 0,036 & 0,096 & 0,743 & 0,132 & 0,308 & \\
\hline & as4 & 0,259 & 0,312 & 0,737 & 0,147 & 0,108 & \\
\hline \multirow{7}{*}{$\begin{array}{l}\text { Exito Empresarial - La percepción de éxito tiene una } \\
\text { influencia positiva en la intención emprendedora }\end{array}$} & ee7 & $-0,065$ & 0,128 & 0,123 & 0,811 & 0,125 & \\
\hline & eе6 & $-0,124$ & 0,189 & 0,135 & 0,728 & 0,076 & \\
\hline & ee3 & $-0,057$ & 0,126 & $-0,066$ & 0,723 & 0,11 & \\
\hline & ee4 & 0,202 & 0,091 & 0,089 & 0,692 & 0,078 & \\
\hline & ee5 & 0,007 & 0,262 & 0,096 & 0,661 & 0,202 & \\
\hline & ee8 & 0,111 & 0,332 & $-0,001$ & 0,638 & 0,285 & \\
\hline & eе2 & 0,304 & 0,163 & 0,134 & 0,634 & 0,23 & \\
\hline \multirow{6}{*}{$\begin{array}{l}\text { Formación y Experiencia -La educación universitaria } \\
\text { fomenta la creación de empresas. }\end{array}$} & ce3 & 0,1 & 0,147 & 0,146 & 0,266 & 0,785 & \multirow[t]{6}{*}{870} \\
\hline & ce4 & 0,134 & 0,144 & 0,105 & 0,183 & 0,784 & \\
\hline & ce5 & 0,08 & 0,158 & 0,334 & $-0,04$ & 0,766 & \\
\hline & ce6 & 0,229 & 0,198 & 0,092 & 0,274 & 0,737 & \\
\hline & cel & 0,114 & 0,234 & 0,31 & 0,17 & 0,674 & \\
\hline & ce2 & 0,112 & 0,062 & 0,244 & 0,184 & 0,605 & \\
\hline
\end{tabular}

Fuente: elaboración propia.

Nota: Método de extracción: Análisis de componentes principales. Método de rotación: Normalización varimax con Kaiser. 
Consecuentemente, con el propósito de dar soporte al cumplimiento del objetivo de esta investigación (analizar si existe una relación entre las variables independientes y la intención emprendedora) se realizó un análisis de correlación de Pearson que permite magnificar la fuerza que existe en la relación de las diversas variables estudiadas. Para ello, cada componente encontrado se transformó en una variable independiente.

Los resultados implican que todas las variables independientes están asociadas de forma positiva y significativa con la intención emprendedora, puesto que los cinco componentes lograron una alta significatividad ( $p$ valor de .000). Finalmente,

\begin{tabular}{|c|c|c|}
\hline \multicolumn{3}{|c|}{$\begin{array}{c}\text { TABLA } 5 \\
\text { ANÁLISIS DE CORRELACIÓN }\end{array}$} \\
\hline \multicolumn{2}{|c|}{ Variables independientes } & \multirow{2}{*}{$\begin{array}{c}\text { Intención } \\
\text { emprendedora } \\
483^{* *}\end{array}$} \\
\hline Valoración & $\begin{array}{l}\text { Correlación de } \\
\text { Pearson }\end{array}$ & \\
\hline & Sig. (bilateral) &, 000 \\
\hline & $\mathrm{N}$ & 143 \\
\hline \multirow[t]{3}{*}{ Formación y experiencia } & $\begin{array}{l}\text { Correlación de } \\
\text { Pearson }\end{array}$ &, $540^{* *}$ \\
\hline & Sig. (bilateral) &, 000 \\
\hline & $\mathrm{N}$ & 143 \\
\hline \multirow[t]{3}{*}{ Conocimiento empresarial } & $\begin{array}{l}\text { Correlación de } \\
\text { Pearson }\end{array}$ &, $382^{* *}$ \\
\hline & Sig. (bilateral) &, 000 \\
\hline & N & 142 \\
\hline \multirow[t]{3}{*}{ Éxito empresarial } & $\begin{array}{l}\text { Correlación de } \\
\text { Pearson }\end{array}$ &, $332^{* *}$ \\
\hline & Sig. (bilateral) &, 000 \\
\hline & N & 143 \\
\hline \multirow[t]{3}{*}{ Crecimiento empresarial } & $\begin{array}{l}\text { Correlación de } \\
\text { Pearson }\end{array}$ &, $629^{* *}$ \\
\hline & Sig. (bilateral) &, 000 \\
\hline & N & 143 \\
\hline
\end{tabular}

Nota: ${ }^{* *}$. La correlación es significativa al nivel 0,01 para poner a prueba cada una de las hipótesis planteadas, se realizó un análisis de regresión múltiple (ver tabla 6).

Al estudiar los ajustes del modelo se puede determinar que este es significativo a través de la prueba de F. Así mismo, la bondad de ajuste asciende a un $48 \%$, lo que llama a un buen poder explicativo del ajuste ya que el restante valor de R2 corresponde a otras variables que no competen a este estudio, de acuerdo con Hair (1999). Los resultados anteriores permiten centrar nuestra atención en las variables de valoración, la percepción de crecimiento y formación universitaria; puesto que las demás variables cuentan con un p valor $>.05$, lo cual significa que no existe evidencia para rechazar la hipótesis nula, la relación entre dichas variables independientes con la intención emprendedora puede no ser parte del modelo. Por lo tanto, se rechazan tanto $\mathrm{H} 3$, como $\mathrm{H} 4$.

Siguiendo el análisis, se determina que la valoración social tiene una influencia positiva y significativa con la Intención Emprendedora, por ello es que se acepta el planteamiento de H1. En este mismo sentido encontramos el crecimiento empresarial, así como la formación y experiencia (relación positiva y significativa), lo cual nos da evidencia suficiente para aceptar tanto $\mathrm{H} 2 \mathrm{como} \mathrm{H} 3$.

Es así como mediante los coeficientes tipificados sostenemos que el Crecimiento Empresarial y la Formación universitaria son las variables que contribuyen en mayor medida a la intención emprendedora, lo cual está asociado a la percepción de éxito y la formación, características intrínsecas de las personas.

\section{CONCLUSIONES}

El propósito de la investigación fue comprobar los factores que impactaban de manera positiva en la intención emprendedora; se encontró que existen factores como las capacidades o habilidades, valoración del emprendedor, conocimientos 


\section{TABLA 6 \\ MODELO DE REGRESIÓN}

\begin{tabular}{|c|c|c|c|c|c|}
\hline \multirow[t]{2}{*}{ Modelo } & \multicolumn{2}{|c|}{$\begin{array}{l}\text { Coeficientes no } \\
\text { estandarizados }\end{array}$} & \multirow[t]{2}{*}{$\begin{array}{c}\text { Coeficientes } \\
\text { tipificados }\end{array}$} & \multirow[t]{2}{*}{$t$} & \multirow[t]{2}{*}{ Sig. } \\
\hline & B & Error típ. & & & \\
\hline (Constante) &, 958 &, 547 & & 1,751 &, 082 \\
\hline Valoración & ,178 &, 069 & ,191 & 2,579 &, 011 \\
\hline Formación &, 347 &, 085 & ,322 & 4,097 &, 000 \\
\hline $\begin{array}{l}\text { Conocimiento } \\
\text { empresarial }\end{array}$ &,- 091 &, 060 &,- 120 & $-1,505$ &, 135 \\
\hline Éxito empresarial &,- 126 & 100 &,- 093 & $-1,257$ &, 211 \\
\hline Crecimiento &, 546 & ,093 & ,493 & 5,896 &, 000 \\
\hline \multirow{4}{*}{ Ajuste del modelo } & $F$ & 27,155 & & & Sig., 000 \\
\hline & R &, 707 & & & \\
\hline & $R^{2}$ &, 500 & & & \\
\hline & $R^{2 \text { CORR }}$ & ,481 & & & \\
\hline
\end{tabular}

Nota: Variable independiente, Intención Emprendedora.

empresariales y objetivos empresariales. Sin embargo, es importante resaltar que la valoración del emprendedor, la educación universitaria y la percepción de desarrollo de la empresa afectan positiva y significativamente la intención emprendedora.

Como sostiene la teoría del comportamiento planeado, la norma subjetiva es importante para cualquier persona, puesto que requiere del reconocimiento social para el desarrollo de sus actividades, lo cual incide si en el entorno se ve de forma positiva a los emprendedores y esto, lo que llevará a un aumento en la creación de empresas.

Dentro de los resultados obtenidos tenemos que la imagen que la sociedad tiene del emprendedor influye en la decisión de iniciar un negocio, en específico la opinión de familia y amigos, lo cual concuerda con los resultados de diversas investigaciones (Fernández, Rueda y Herrero, 2011; Liñan, Nabi y Krueger, 2013; Durán et al., 2016; Osorio y Roldán, 2016; Riquelme et al., 2016).

Así mismo, los universitarios se sienten capacitados para desarrollar habilidades para la creación, desarrollo y mantenimiento de su propia empresa (Bergmann et al, 2016; Hoppe, 2016; Rachmawan, Lizar y Mangundjaya ,2015). De igual manera, la percepción de éxito personal, en la forma de desarrollo de la empresa juega un rol importante en el desarrollo del emprendimiento en universitarios (Fernández y Gervilla, 2013; Liñan, Nabi y Krueger, 2013; Mora, 2011).

Finalmente, es relevante mencionar que dichos resultados se limitan a estudiantes de carreras económico administrativas de instituciones de educación superior en Ciudad Victoria, Tamaulipas. Lo anterior genera interés en replicar el estudio 
enfocado en otras formaciones profesionales y en un ámbito geográfico distinto para poder establecer semejanzas, diferencias y particularidades.

\section{REFERENCIAS}

Ajzen, I. (1991). The theory of planned behavior. Organizational Behavior and Human Decision Processes, 50, 179-211.

Ajzen, I. (2002). Perceived Behavioral Control, Self-efficacy, Locus of Control and the Theory of Planned Behavior. Journal of Applied Social Psychology, $32,1-20$.

Aloulou, W. y Aloulou, J. (2016). Predicting entrepreneurial intentions of final year Saudi university business students by applying the theory of planned behavior. Journal of Small Business and Enterprise Development, 23(4), 1142-1164.

Amit, R., Glosten L. y Muller, E. (1993). Challenges to theory development in entrepreneurship research. Journal of Management Studies, 30(5), 815-834

Arias, A. V., Cadavid, L., Echeverri, D. y Awad, G. (2012). Factores que inciden en las intenciones emprendedoras de los estudiantes. Revista Venezolana de Gerencia, 17(57).

Audretsch, D. y Keilbach, M. (2004). Does entrepreneurship capital matter? Entrep. Theory Pract, 419-429.

Bandura, A. (1997). Self-efficacy: The exercise of control. Estados Unidos: Macmillan.

Bandura, A., Freeman, W. y Lightsey, R. (1999). Self-efficacy: The exercise of control. Journal of Cognitive Psychotherapy, 13(2), 158-166.

Bergmann, H., Hundt, C. y Sternberg, R. (2016). What makes student entrepreneurs? On the relevance (and irrelevance) of the university and the regional context for student start-ups. Small Business Economics, 47(1), 53-76.

Bird, B. (1988). Implementing Entrepreneurial Ideas: The Case for Intentions. Academy of Management Review, 13(3), 442-453.
Boh, W., De-Haan, U. y Strom, R. (2016). University technology transfer through entrepreneurship: faculty and students in spinoffs. The Journal of Technology Transfer, 41(4), 661-669.

Brito, M., Cruz, A. y Hernández, A. (2014). Un paso más en la investigación de la intención emprendedora del estudiante universitario: GUESSS. Revista de Estudios Empresariales, segunda época (2), 63-80.

Brockhaus, R.H. (1982). The psychology of entrepreneur. En C.A. Kent, D.L. Sexton y K.H. Vesper (eds.). Encyclopedia of entrepreneurship (39-56). Englewood Cliffs NJ: Prentice Hall.

Cantillon, R. (2001). Essays on the nature of commerce in general. Estados Unidos: Transaction Publishers.

Cantner, U., Goethner, M. y Silbereisen, R. K. (2017). Schumpeter's entrepreneur-A rare case. Journal of Evolutionary Economics, 27(1), 187-214.

Carsrud A.L. y Johnson R.W. (1989). Entrepreneurship: A Social Psychological Perspective. Entrepreneurship \& Regional Development, 1, p.21-31.

Casson, M. (1982). The Entrepreneur: An Economic Theory, Estados Unidos: Barnes \& Noble Books.

Collins O., Moore D. y Unwalla D. (1964). The enterprising man. Estados Unidos: MSU Business Studies, 254p.

Davids, L. E. (1963). Characteristics of Small Business Founders in Texas and Georgia. Bureau of Business Research University of Georgia.

Díaz, C. (2002). La Creación de Empresas: Revisión Histórica de Teorías y Escuelas. España: Ediciones La Coria. Fundación Xavier de Salas.

Durán, B., Martínez, J., López, J., Flores, G. y Jiménez, G. (2016). El desarrollo de la creatividad como estrategia para fomentar la apropiación de las TIC en la docencia universitaria. XIX Congreso Internacional EDUTEC 2016.

Edelman, L., Manolova, T., Shirokova, G. y Tsukanova, T. (2016). The impact of family support on young entrepreneurs' start-up activities. Journal of Business Venturing, 31 (4), 428-448. 
Engle, R. L., Schlaegel, C.y Dimitriadi, N. (2011). Institutions and entrepreneurial intent: A cross-country study. Journal of Developmental Entrepreneurship, 16(02), 227-250.

Evans, D. y Leighton L., (1990). Small Business Formation by Unemployed and Employed Workers, Small Business Economics, 2(4), pp. 319-330.

Fernández, R. y Gervilla, M. (2013). Factores explicativos de la intención de emprender en la mujer. Aspectos diferenciales en la población universitaria según la variable género. Cuadernos de gestión, 13(1), 127.

Fernández, A., Rueda, M. y Herrero, Á. (2011). Estudio de la actitud emprendedora de los estudiantes universitarios de la facultad de CC. EE. y empresariales de Cantabria. XVI Congreso de la Asociación Española de Contabilidad y Administración de Empresas (AECA), pp. 1-22.

Fernández, J. y Romero, I. (2013). Entrepreneurial quality and regional development: Characterizing SME sectors in low income areas. Papers in Regional Science, 92(3), 495-513. Recuperado de: https://doi.org/10.1111/j.1435-5957.2012.00421.x

Forsström, H., Jussila, I. y Kolhinen, J. (2015). Business school students' social construction of entrepreneurship: Claiming space for collective entrepreneurship discourses. Scandinavian Journal of Management, 31(1), 102-120.

Gartner, W.B. (1988): Who IS an Entrepreneur? Is the Wron Question. American Journal of Small Business, 12(4), 11-32.

Guerrero, M. y Urbano, D. (2012). The development of an entrepreneurial university. The journal of technology transfer, 37(1), 43-74.

Hair, J. (1999). Análisis multivariante. Estados Unidos: Pretice-Hall

Hamilton, B. (2000). Does entrepreneurship pay? An empirical analysis of the returns to selfemployment. Journal of Political Economy, 108(3), 604-631.

Hayter, C. S. (2016). A trajectory of early-stage spinoff success: the role of knowledge intermediaries within an entrepreneurial university ecosystem. Small Business Economics, 47(3), 633-656.

Hernández, K., González, L. y Herrera, M. (2015). Global Entrepreneurship Monitor: Reporte Nacional de México. México: Instituto Tecnológico de Monterrey.

Hofstede, G., Noorderhaven, N., Thurik, A., Uhlaner, L.,Wennekers, A. y Wildeman, R. (2004). Culture's role in entrepreneurship: Self-employment out of dissatisfaction. Estados Unidos: Edward Elgar.

Hoppe, M. (2016). Policy and entrepreneurship education. Small Business Economics, 46(1), 13-29.

Karimi, S., Biemans, H. J., Lans, T., Chizari, M. y Mulder, M. (2016). The Impact of entrepreneurship education: A study of Iranian students' entrepreneurial intentions and opportunity identification. Journal of Small Business Management, 54(1), 187-209.

Lee, L., y Wong, P. (2004). Attitude towards entrepreneurship education and new venture creation. Journal of Enterprising Culture, 11, 339-357.

Liñan, F. (2004). Intention-based models of entrepreneurship education. Piccola Impresa/Small Business, 2004(3), 11-35.

Liñan, F. y Chen, Y. W. (2009). Development and Cross Cultural application of a specific instrument to measure entrepreneurial intentions. Entrepreneurship theory and practice, 33(3), 593-617.

Liñan, F., Nabi, G. y Krueger, N. (2013). British and Spanish Entrepreneurial Intentions: A Comparative Study. Revista de economía Mundial, 33.

Lucas, W, Cooper, S, Ward, T. y Cave, F. (2009). Industry placement, authentic experience and the development of venturing and technology selfefficacy. Technovation, 29 (11), 738-752.

MacMillan, I. C. (1986). To really learn about entrepreneurship, let's study habitual entrepreneurs. Journal of Business Venturing, 1, 241-243.

Martin, B., McNally, J. y Kay, M. (2013). Examining the formation of human capital in entrepreneurship: A meta-analysis of entrepreneurship education outcomes. Journal of Business Venturing, 28(2), 211-224. 
McClelland, D. C. (1961). The Achieving Society. Princeton: D. Van Nostrand Company.

Micozzi, A., Micozzi, A., Lucarelli, C. y Lucarelli, C. (2016). Heterogeneity in entrepreneurial intent: the role of gender across countries. International Journal of Gender and Entrepreneurship, 8(2), 173-194.

Mills, D. y Schuman, L. (1985). Industry Structure with Fluctuating Demand, American Economic Review, 75(4), septiembre, pp. 758-767.

Mora, R. (2011). Estudio de actitudes emprendedoras con profesionales que crearon empresa. Revista escolar de administración y negocios, 71, pp. 70-83.

Mueller, S. y Thomas, A. (2001). Culture and entrepreneurial potential: A nine country study of locus of control and innovativeness. Journal of Business Venturing, 16(1), 51-75.

Nueno, P. (1994). Emprendiendo: el arte de crear empresas y sus artistas. España: Ediciones Deusto.

Osorio, F. y Roldán, J. C. L. (2016). Intención emprendedora de estudiantes de educación media: extendiendo la teoría de comportamiento planificado mediante el efecto exposición. Cuadernos de Administración, 28(51), 103-131.

Pérez, C., González, M. y Rodríguez, L. (2016). Competencies for entrepreneurship as a career option in a challenging employment environment. Career Development International, 21(3), 214-229.

Plotnikova, M., Romero, l. y Martínez, J. (2016). Process innovation in small businesses: the self-employed as entrepreneurs. Small Business Economics, 47(4), 939-954.

Rachmawan, A., Lizar, A. y Mangundjaya, W. (2015). The role of parent's influence and self-efficacy on entrepreneurial intention. The Journal of Developing Areas, 49(3), 417-430.

Riquelme, E., Ortiz, M., Álvarez, M. y Baro, C. (2016). Estudio comparativo de las intenciones emprendedoras en estudiantes universitarios: caso práctico Ingeniería Civil Industrial, Universidad Arturo Prat. Ciencia Amazónica (lquitos), 6(1), 48-62.
Romero, A. y Milone, M. (2016). El Emprendimiento en España: Intención 95 Emprendedora, Motivaciones y Obstáculos. Revista de Globalización, Competitividad y Gobernabilidad, 10(1), 95.

Schumpeter, J. (1934). The theory of Economic DeveIopment: An Inquiry into Profits, Capital, Credit, Interest and the Business Cycle. Estados Unidos: Harvard University Press.

Shane, S. y Eckhardt, J. (2003). The individual-opportunity Nexus. En Acs, Z.J. \& Audretsch D.B. (eds.), Handbook of Entrepreneurship Research: An Interdisciplinary Survery and Introduction. Estados Unidos: Kluwe Academic Publishers.

Shane, S. (2004). Academic Entrepreneurship: University Spinoffs and Wealth Creation. Chelltenham, Reino Unido: Edward Elgar.

Shapero, A. y Sokol, L. (1982). The social dimensions of entrepreneurship. Estados Unidos: Encyclopedia of entrepreneurship Englewood Cliffs,

Staniewski, M. y Awruk, K. (2016). Start-up intentions of potential entrepreneurs-the contribution of hope to success. Ekonomska istraživanja, 29(1), 233-249.

Steyaert, C. y Katz, J. (2004). Reclaiming the space of entrepreneurship in society: geographical, discursive and social dimensions. Entrepreneurship \& Regional Development, 16(3), p.179-196.

Villasana, M., Alcaraz, R. y Álvarez, M. (2016). Examining entrepreneurial attributes of Latin American female university students. Gender and Education, 28(1), 148-166.

Weiss, L. W. (1991) Structure, Conduct and Performance. Audretsch, D. y Hideki Y. (eds.). Estados Unidos: New York University Press.

Wennekers, S., van Stel, A., Thurik, R. y Reynolds, P. (2005). Nascent entrepreneurship and the level of economic development. Small Business Economics, 24(3), 293-309.

Recibido: 10 de enero 2017

Aceptado:30 de junio 2017 
This document was created with Win2PDF available at http://www.win2pdf.com. The unregistered version of Win2PDF is for evaluation or non-commercial use only. This page will not be added after purchasing Win2PDF. 